\title{
Inovação na Estratégia da Saúde Social no Envelhecimento: Papel da Economia Social em Resposta à Pandemia
}

\author{
Innovation in the Social Health Strategy in Aging: Role of the Social Economy in \\ Response to the Pandemic
}

\section{Mariana Gomes}

Santa Casa da Misericórdia de Marco Canaveses, Portugal mariana.gomes85@gmail.com

\section{Lília Pinto}

Santa Casa da Misericórdia de Marco Canaveses, Portugal liliapinto2010@gmail.com

\section{Maria Amélia Ferreira}

Santa Casa da Misericórdia de Marco Canaveses, Departamento de Ciências da Saúde Pública e Forenses e Educação de Medicina da Universidade do Porto, Portugal

Conflito de interesses: nada a declarar. Financiamento: Este programa obteve financiamento para a sua execução da SCMMC e de Prémios como, BPI "La Caixa" Seniores 2020, BPI "La Caixa” Rural 2019, Fidelidade Comunidade 2018, Fundação Calouste Gulbenkian - Envelhecimento na Comunidade.

Data de Submissão: 14/05/2021

Data de Aprovação: 29/07/2021

Todo o conteúdo do JIM é licenciado sob Creative Commons, a menos que especificado de outra forma e em conteúdo 


\section{RESUM0:}

0 complexo processo de envelhecimento pensado à luz da crise pandémica, que abalou 0 funcionamento de toda a sociedade, impulsionou abordagens distintivas à prestação de cuidados de saúde no envelhecimento. A Santa Casa da Misericórdia do Marco de Canaveses (SCMMC), no âmbito da Economia Social e em resposta ao desafio do futuro na saúde social no envelhecimento, através da articulação indissociável da saúde e da área social, inovou na humanização e disponibilização de cuidados de saúde especializados na população sénior, centrados na pessoa e baseados na relação. Através da implementação de diferentes projetos, surge uma perspetiva de inovação, comunicação e empoderamento. No decurso da sua execução - com o apoio a tecnologias de comunicação (teleconsulta) - surgirá uma abordagem integrativa no binómio saúde-social, criando um planeamento adaptativo e um desenvolvimento evolutivo, personalizado à situação da pessoa idosa, de aplicação célere e desenvolvimento contínuo.

Palavras-chave: Economia Social, Saúde Social, Envelhecimento, Teleconsulta

\section{ABSTRACT:}

The complex aging process approached within the scope of the pandemic crisis, which disrupted the functioning of the whole society, has enhanced distintive approaches to health care strategies in aging. The Holy Mercy House of Marco de Canaveses (SCMMC), a Social Economy Institution, in response to the future challenges in social health in aging, through the joint articulation of health/social care, has provided a set of innovation in the humanization and availability of specialized heath care for the senior population, centered on the person and based on the relationship. Through different projects under development, a perspective of innovation, communication and empowerment emerges, supported by the use of new technologies (teleconsultation). During its course, it will allow an integrative approach in health-social responses, creating adaptive planning and an evolutionary development, personalized to the situation of the old person, providing an adequate time-schedule application and long-term development.

Keyword: Social Economy, Social Health, Aging, Teleconsultation 
https://doi.org/10.29073/jim.v2i2.418

\section{ENQUADRAMENTO}

0 envelhecimento da população está a avançar rapidamente em todo o mundo (OMS, 2015) marcando uma tendência progressiva e inalterável nas próximas décadas, pelo que é fundamental que as sociedades estejam estruturadas para atender uma população envelhecida (OMS, 2021). Segundo o Instituto Nacional de Estatística (2015) prevê-se que a proporção mundial de pessoas com 60 ou mais anos de idade possa atingir 21,1\% em 2050. Portugal, à semelhança dos restantes países da Europa, tem vindo a demonstrar profundas alterações demográficas, relacionadas essencialmente com o aumento da longevidade e da população idosa, e com a diminuição da natalidade e população jovem (DGS, 2017), colocando-se numa das posições com maior número de pessoas idosas por cada 100 jovens (Marques, 2020).

0 atual contexto pandémico, provocado pelo Coronavírus SARS-Cov2 (COVID-19), emerge em pleno processo de envelhecimento da população e revela-se especialmente impactante por todas as suas repercussões, nomeadamente, a incapacidade de resposta dos serviços de saúde nos casos urgentes e doenças crónicas, com efeitos devastadores ao nível da perda de vidas (Melo, Tavares \& Duarte, 2020). Em Portugal, as medidas adotadas de contenção e mitigação da pandemia, impuseram um isolamento com especificidades acrescidas para as pessoas idosas, 0 qual deve ser mais restrito e o mais longo possível (Henrique \& Dias 2020), em grande medida por esta população apresentar maior vulnerabilidade decorrente da senescência ou senilidade (Marques, 2020).

Assim, as constantes mudanças do perfil das pessoas idosas exigem uma visão e ponderação pluridimensional na sua abordagem na área da saúde, de modo a proporcionar-Ihes uma vida feliz, saudável, com qualidade e bem-estar, numa ótica de envelhecimento saudável (Vara, 2012). Reveste-se de extremo significado garantir que as pessoas idosas vivam mais anos, mas sobretudo que estes anos sejam plenos de saúde física e integridade mental (Rosa, 2020b). Aliás, para autores como Castro, Will, Castro, Ximenes e Cordeiro (2020), envelhecer de forma saudável é ter capacidade de aceitar todas as mudanças fisiológicas resultantes da idade e gerir as doenças e limitações subjacentes, de modo a que estas não impeçam a experiência pessoal de um envelhecimento bem-sucedido.

Como mostra a evidência, a maioria das pessoas prefere envelhecer em casa e na comunidade, no seu meio sócio familiar (Wiles, Leibing, Guberman, Reeve \& Allen, 2011; WHO, 2016), viver com segurança e de forma independente, o que sustenta cada vez mais a intervenção Ageing in Place (Marques, 2018; Fonseca, 2018). Nesta consonância e atendendo ao meio rural importa 
salientar que o envelhecimento rural diz respeito às implicações do envelhecimento, padrões, processos e a todos os resultados inerentes, nas áreas rurais (Currie \& Philip, 2019). Porém, torna-se um desafio incontornável à sociedade não só pela diversidade desta população, mas também pelas suas idiossincrasias (composição familiar, estruturas económicas e estado de saúde), implementar um conjunto de intervenções de caráter integrado nas áreas da saúde-social que desenvolvam ambientes promotores do envelhecimento saudável na comunidade (Rosa, 2020a).

Retomando, a crise sanitária inaudita e os muitos estudos já realizados, percebe-se que a infeção provocada pela COVID-19 encontra neste grupo etário, mais fragilizado, empobrecido e isolado, um campo mais vulnerável, condicionado por um sistema imunológico mais debilitado, o que associado a comorbilidades frequentes, aumenta significativamente o risco de morte (Henrique \& Dias 2020). Há evidências de que um dos impactos da COVID, no que se refere ao isolamento social na população sénior, se relaciona com o aumento dos sentimentos de solidão, tédio, desesperança e depressão (Silva, Viana \& Lima, 2020). Logo, idosos mais isolados, com uma rede de suporte social mais restrita, identificam experiências mais negativas no acesso a cuidados de saúde primários (Pimentel, Fernandes, Carlos \& Bastos, 2019). A necessidade de distanciamento físico impôs alterações drásticas nos sistemas de saúde, com medidas específicas ao nível dos atendimentos presenciais, quer por ausência de capacidade quer para evitar a disseminação da doença (Almeida, Coelho, Martins \& Guarda, 2020) e criou uma oportunidade para a adoção de soluções de cuidados remotos digitais - teleconsulta - capazes de garantir a disponibilidade assistencial contínua e o cuidado ao doente não-COVID (Oliveira, Queirós, Gomes \& Monteiro., 2020). A passagem para soluções digitais de saúde perpetuou-se assim como condição necessária para atingir padrões mais altos de qualidade de saúde, em tempos de pandemia (Vaz Almeida, 2020), para além de facilitar o acesso, o acompanhamento continuado e a proatividade dos cuidados. Permite o exercício de uma medicina preventiva, preditiva e personalizada, com resultados significativos de eficiência e qualidade (Oliveira et al., 2020). Todavia, a teleconsulta apresenta um custo acrescido, relativo ao acesso a equipamentos e condições de conetividade, o que se torna mais expressivo na população idosa, com menor literacia tecnológica e de saúde (Espanha, 2013). Aliás, uma grande proporção de níveis de literacia em saúde problemáticos e inadequados, regista-se na população idosa, representando 80\% entre as pessoas com mais de 75 anos (Espanha, Ávila \& Mendes, 2016). Ainda neste campo, se se considerar que o acesso, a compreensão e o uso da informação em saúde são por si só complexos percebe-se que é importante apoiar a pessoa e a família que a acompanha, de modo a que consigam passar as barreiras que se levantam, nas várias dimensões cognitivas inerentes 
ao sistema de teleconsulta (Vaz de Almeida, 2021a). Estudos indicam que um bom acesso, uma boa compreensão e uso da informação e dos serviços, melhora resultados em saúde ao longo do ciclo de vida (Vaz de Almeida, 2021b). Neste sentido, é fundamental o envolvimento dos familiares da pessoa idosa nas ações de promoção da literacia em saúde e de mudança de comportamentos de saúde, uma vez que, em questões de saúde, estes são considerados importantes fontes de suporte e elementos influentes em todo o processo de tomada de decisão (DGS, 2019). Ora, é no seio da família onde ocorre grande parte dos processos de reciprocidade de interesses, relações e serviços, isto é, a chamada solidariedade (Pimentel et al. 2019).

\section{OBJETIVOS E DESTINATÁRIOS}

Com a implementação de uma estratégia sistemática de abordagem multidisciplinar procura-se aumentar 0 acesso a cuidados de saúde especializados, reduzir a prevalência, adiar 0 aparecimento, controlar o agravamento e o impacto das doenças crónicas, potenciar 0 envelhecimento no domicílio, promover a humanização dos cuidados de saúde, melhorar o bemestar e qualidade de vida, e reduzir o sofrimento psíquico e o sentimento de solidão.

Os projetos desenvolvidos pela SCMMC destinam-se a pessoas com mais de 65 anos, a residir no domicílio, em zonas rurais do Concelho do Marco de Canaveses, com escasso apoio social e económico, com dificuldade na gestão da doença crónica e ausência de suporte familiar. Indiretamente também deles beneficiam os familiares e/ou cuidadores das pessoas idosas, nomeadamente ao nível da psicoeducação, com disponibilização de informações específicas sobre as temáticas da saúde, e de competências relacionadas com a teleconsulta. Esta abordagem é precursora do desenvolvimento de um modelo integrado de saúde social no envelhecimento (MISSE), como corolário das intervenções instituídas nesta população.

\section{METODOLOGIA}

Os Projetos foram pensados no âmbito de candidaturas nas áreas da saúde social, promovidas por diferentes entidades (e.g. Prémios: BPI "La Caixa” Seniores 2020, BPI "La Caixa” Rural 2019, Fidelidade Comunidade 2018, Fundação Calouste Gulbenkian - Envelhecimento na Comunidade) e levadas a cabo pela SCMMC - uma entidade da Economia Social que atua em meio rural, com intervenção na saúde e na ação social. Estes representam o desafio da intervenção no envelhecimento e foram estruturados com a finalidade de se construir um modelo de prestação humanizada de cuidados de saúde no envelhecimento, no domicílio - Serviço Móvel de Saúde 
(SMS), Projeto SMS+Cuidadores, Projeto Rural SMS2Care, Projeto SMS+GreenCare e concretizando-se no desenvolvimento de um Modelo Integrado de Saúde Social no Envelhecimento (MISSE).

Os Projetos integram o SMS, o corpo clínico especializado, do Hospital de Santa Isabel, e as tecnologias de comunicação - teleconsulta - de modo a garantir o maior número de acesso aos cuidados de saúde especializados. A SCMMC disponibiliza meios de acesso remoto, especificamente Tablet's, assim como a formação e apoio necessários, aos utentes e familiares, cuidadores ou voluntários (pessoas disponíveis na comunidade para prestar este apoio) sobre todo o processo da teleconsulta.

A avaliação dos indicadores será concretizada em dois momentos: pré e pós intervenção, com o recurso ao Questionário de Qualidade de Vida e Saúde (EUROHIS - QOL-8), à Escala de Solidão (UCLA) e à Escala de Depressão Geriátrica (GDS -15). A avaliação da Satisfação dos Utentes com os Cuidados de Saúde será avaliada a partir do Questionário EUROPEP - European Task Force on Patient Evaluation of General Pratice Care (versão portuguesa). A avaliação das consultas de especialidade será aferida pelo número de consultas realizadas no domicílio, nas instalações da SCMMC e por teleconsulta.

\section{RESULTADOS ESPERADOS}

Com a implementação desta intervenção integrada prevê-se que a SCMMC possa liderar a intervenção ao nível das boas-práticas no "ageing in place", constituir-se como um referencial de humanização em saúde social e possibilitar o envolvimento integrado do corpo clínico do Hospital, numa ótica sistémica e de proximidade.

Estima-se que se irá contribuir para uma diminuição do acesso ao Serviço Nacional de Saúde, que apresenta uma resposta já insuficiente. Prevê-se ainda que, para os resultados diretos, se realizem em média 3 consultas de especialidade, apoio por teleconsulta, aumentar em $20 \%$ o acesso a cuidados de saúde especializados, melhorar o bem-estar e qualidade de vida pelo menos em $75 \%$, e reduzir o sofrimento psíquico e o sentimento de solidão em $50 \%$.

Ao nível de efeitos na comunidade prevê-se aumentar a oferta de resposta de saúde especializada para os idosos com doença crónica, criar um maior sentido de comunidade, aumentar os níveis de literacia em saúde com a criação e divulgação de flyers informativos.

Para promover estas respostas, a SCMMC estabeleceu uma nova parceria, com a PRIMEDEV, para o desenvolvimento de uma APP de teleconsulta, articulada com o processo clínico 
https://doi.org/10.29073/jim.v2i2.418

informático, o que constituiu uma mais valia potenciando o apoio à distância, reduzindo os custos com deslocações e aumentando a segurança na prestação de cuidados de saúde.

\section{CONCLUSÃO}

Esta estratégia de intervenção, delineada para uma faixa cada vez mais expressiva da população, as pessoas idosas, constitui uma inovação na abordagem da saúde social no envelhecimento, tendo como protagonista a Economia Social. Se por si só o processo de envelhecimento é complexo e tem motivado preocupação por parte das Instituições Nacionais e Internacionais, em pleno contexto pandémico tem revelado efeitos claramente devastadores. Nesta consonância, enquanto que promover a qualidade de vida é a tónica dominante do envelhecimento ativo, humanizar os cuidados de saúde e a proximidade com os sistemas de saúde constituem o desafio do futuro para um envelhecimento saudável.

Com este trabalho multidisciplinar procuramos não só acelerar a transição para novos paradigmas digitais de prestação de cuidados de saúde mas, acima de tudo, consubstanciar esta experiência numa realidade consolidada, após a crise pandémica, num esforço de constante inovação, otimização e integração, num meio rural empobrecido e envelhecido A par desta motivação procuramos despertar para a crescente complexidade da realidade social, mover a esfera social e familiar, e ser promotores da educação para a saúde, apelando para a necessidade de se atingir níveis de literacia tais, que permitam a utilização concreta das tecnologias e uma compreensão clara da informação de saúde. Este parece-nos o caminho para o sucesso da saúde social no envelhecimento. 
https://doi.org/10.29073/jim.v2i2.418

\section{BIBLIOGRAFIA}

Almeida, C.V., Coelho, I., Martins, P., \& Guarda, L. (2021). Saúde Digital em tempos de pandemia: Encontrar o sentido do espaço, comunicação e proximidade da saúde face-a-face, respeitando as diferenças. Associação Portuguesa para a Promoção da Saúde Pública (APPSP). [online]. Disponível em: www.appsp.pt

Castro, A. P., Will, G.B., Castro, M.R., Ximenes, C. F., \& Cordeiro, M.S. (2020). Vivendo em comunidade, envelhecendo de forma saudável. Revista Eletrónica Trimestral de Enfermería. Disponível em: http://scielo.isciii.es/pdf/eg/v19n57/pt_1695-6141-eg-19-57-302.pdf

Currie, M. \& Philip, L. (2019). Rural Ageing. Encyclopedia of Gerontology and Population Aging. Disponível em: https://www.researchgate.net/publication/335272764_Rural Ageing

Direção-Geral da Saúde (2017). Estratégia Nacional Para o Envelhecimento Ativo e Saudável 20172025. Lisboa: DGS.

Direção-Geral da Saúde. (2019a). Manual de boas práticas literacia em saúde: Capacitação dos profissionais de saúde. Lisboa: Autor.

Espanha, R. (2013). Informação e Saúde. Fundação Francisco Manuel dos Santos. Disponível em: https://www.ffms.pt/publicacoes/detalhe/846/informacao-e-saude

Espanha, R., Ávila, P. \& Mendes, R. V. (2016). Literacia em saúde em Portugal: Relatório síntese. Lisboa: Fundação Calouste Gulbenkian.

Fonseca, A. (2018). Boas práticas de Ageing in Place: Divulgar para valorizar, Guia de boas práticas. Disponível em: https://content.gulbenkian.pt/wpcontent/uploads/2018/05/15122919/ageing in place web.pdf

Henriques, A., \& Dias, I. (2020). Da emergência de um novo vírus humano à disseminação global de uma nova doença - Doença por Coronavírus 2019 (COVID-19) As duas faces do isolamento dos idosos em tempo de pandemia: quem "achata a curva" da solidão? Disponível em: https://hdl.handle.net/10216/131205

Instituto Nacional de Estatística. (2015). Estatísticas de Portugal. Disponível em: https://www.ine.pt/xportal/xmain?xpgid=ine main\&xpid=INE\&xlang=pt.

Marques, R. A. D. (2018). Ageing in Place: Estratégias para Envelhecer no Domicílio. Instituto Superior de Ciências Sociais e Políticas Universidade de Lisboa. Lisboa. Disponível em: http://hdl.handle.net/10400.5/16454

Marques, D. (2020). Solidão em Tempos de COVID. Instituto Miguel Torga, Disponível em: http://repositorio.ismt.pt/jspui/bitstream/123456789/1237/1/Disserta\%C3\%A7\%C3\%A30\%2 0de\%20Mestrado.pdf 
Melo, R. B., Tavares, N. T., \& Duarte, R. (2020). COVID-19 e os Danos Invisíveis. Acta Médica Portuguesa, 33(5), 293-294.

Oliveira, V.P., Queirós, L., Gomes, P. P., \& Monteiro, M.S. (2020). COVID-19 e a Transformação Digital dos Cuidados de Saúde: "A Pastilha de Mentos na Coca-Cola Diet". Gazeta Médica, $2(7), 124-131$.

Organização Mundial de Saúde. (2015). Resumo: Relatório mundial de envelhecimento e saúde. Disponível em:

https://apps.who.int/iris/bitstream/handle/10665/186468/WHO FWC ALC 15.01_por.pdf\%3Bjse

Organização Mundial de Saúde. (2021). Década do envelhecimento saudável 2020-2030. Disponível em: https://www.paho.org/pt/decada-do-envelhecimento-saudavel-2020-2030

Pimentel, M. H, Fernandes, H., Carlos, M.F.A., \& Bastos, M. A. M. S. C. (2019). Importance of social network for the successful aging and health, of the elderly. Journal of Aging \& Innovation, 8 (1), 68-84.

Rosa, M. J. V. (2020a). Envelhecimento Demográfico em Fase de COVID-19. Artigo de opinião em Revista da Sociedade Portuguesa de Medicina Interna, 27-30.

Rosa, M.J.V. (2020b). Um Tempo sem Idades. Ensaio sobre o Envelhecimento da População. Edições Tinta da China.

Silva, M. L., Viana, S. \& Lima, P.T. (2020). Impacto na saúde mental do idoso durante o período de isolamento social em virtude da disseminação da doença COVID19: uma revisão literária. Revista Diálogos em Saúde, 3 (1).

Vara, M. (2012). 0 olhar do gerontólogo. In F. Pereira (Coord.), Teoria e prática da gerontologia (pp. 65-67). Viseu: PsicoSoma.

Vaz de Almeida, C. (2020). Organizações e profissionais de saúde: e depois continuamos no digital? A Pátria. Disponível em: https://apatria.org/autor/vazdealmeidacristinagmail-com

Vaz de Almeida, C. (2021a). Telesaúde: os olhos da saúde. A Pátria. Disponível em: https://apatria.org/saude/telesaude-os-olhos-da-saude/

Vaz de Almeida, C. (2021b). Não basta oferecer ipads! A literacia em saúde exige mais. A Pátria. Disponível em: https://apatria.org/ciencias/nao-basta-oferecer-ipads-a-literacia-em-saudeexige-mais/

Wiles, J.L., Leibing, A., Guberman, N., Reeve, J. \& Allen, R. E. (2012). The meaning of "aging in place" to older people. The Gerontologist, 52 (3), 357-366. Disponível em: https://doi.org/10.1093/geront/gnr098 
https://doi.org/10.29073/jim.v2i2.418

World Health Organization. (2016). The Global strategy and action plan on ageing and health. World Health Organization. Disponível em: https://www.who.int/ageing/WHO-GSAP-2017.pdf?ua=1 